\title{
Meso-Scale Transmission Electron Microscope Tomography Applied for Wax Distribution in Toner Particles
}

\author{
Mino Yang,,${ }^{1,2}$ Jun-Ho Lee, ${ }^{1,2}$ Hee-Goo Kim, ${ }^{1}$ Euna Kim, ${ }^{1}$ Young-Nam Kwon, ${ }^{1}$ Jin-Gyu Kim, ${ }^{3}$ \\ and Cheol-Woong Yang ${ }^{2, \star}$ \\ ${ }^{1}$ Analytical Engineering Group, Samsung Advanced Institute of Technology (SAIT), Youngin, Gyeonggi-do 446-712, Korea \\ ${ }^{2}$ School of Advanced Materials Science \& Engineering, Sungkyunkwan University, Suwon, Gyeonggi-do 440-746, Korea \\ ${ }^{3}$ Division of Electron Microscopic Research, Korea Basic Science Institute (KBSI), Yuseong-gu, Daejeon 305-806, Korea
}

\begin{abstract}
Distribution of wax in laser printer toner was observed using an ultra-high-voltage (UHV) and a medium-voltage transmission electron microscope (TEM). As the radius of the wax spans a hundred to greater than a thousand nanometers, its three-dimensional recognition via TEM requires large depth of focus (DOF) for a volumetric specimen. A tomogram with a series of the captured images would allow the determination of their spatial distribution. In this study, bright-field (BF) images acquired with UHV-TEM at a high tilt angle prevented the construction of the tomogram. Conversely, the Z-contrast images acquired by the mediumvoltage TEM produced a successful tomogram. The spatial resolution for both is discussed, illustrating that the image degradation was primarily caused by beam divergence of the Z-contrast image and the combination of DOF and chromatic aberration of the BF image from the UHV-TEM.
\end{abstract}

Key words: toner, ultra-high-voltage TEM, STEM, tomography, bright field, depth of focus, Z-contrast

\section{INTRODUCTION}

Printing is one of the largest industries whose profit mostly comes from consumables, such as toner. As a result, the development on high-quality toner attracts industrial interest and is devoted to controlling the distribution of wax in toner particles. Toner is primarily a mixture of resin and wax, with a small quantity of pigment. Wax acts to fix the toner to the paper at the final stage of printing. A fuser heated to $\sim 170^{\circ} \mathrm{C}$ melts the wax and presses the toner to tightly adhere to the paper in a short period of time. In the process, molten wax is simultaneously leaked out of the toner. The energy consumption and printing quality rely on the wax dispersion inside the toner (Maruta, 1997). Therefore, microscopic investigation of the toner is strongly required, which provides information on the size, number, and distribution of the wax.

The resolution in a transmission electron microscope (TEM) image is normally treated with electron scattering in the electron beam-specimen interaction volume, which requires a thinner specimen and higher electron beam energy that effectively reduce beam spread $(\boldsymbol{b})$ and chromatic aberration $\left(C_{c}\right)$ to minimize their effect on the image. However, for the investigation of wax dispersed in a micrometer-sized toner particle spanning a hundred to more than a thousand nanometers in diameter, relatively thick TEM specimens are required to produce bulky images containing three-dimensional information. In addition, the specimen tilt for a tomogram significantly increases its virtual thickness (total $\mathrm{Z}$ height occupied by the specimen

() MICROSCOPY SOCIETY OF AMERICA 2013

${ }^{\star}$ Corresponding author. E-mail: cwyang@skku.edu in the TEM column) proportional to $\sin ^{-1} \theta$. The radius of the toner particles was $4-8 \mu \mathrm{m}$. Such a thick virtual thickness is inadequate for the magnification range of TEM, thus requiring ultra-high-voltage (UHV)-TEM comprising of a large depth of field (DOF) or high-angle annular dark-field (HAADF) scanning TEM (STEM) of medium voltage that, to date, cannot be used in a UHV-TEM (Williams \& Carter, 1996). On the basis of an experimental comparison of the image resolution of a thick specimen at a given voltage, STEM produces the same resolution as a conventional TEM from a sample five times thicker (Cosslett, 1979).

In this study, we compared TEM tomography lying in the range of over hundred nanometer scale with $1.25 \mathrm{MeV}$ TEM in bright-field (BF) mode and $200 \mathrm{keV}$ TEM in HAADF-STEM mode. The spatial resolution for both is discussed in terms of beam divergence, DOF, and chromatic aberration.

\section{Materials and Methods}

A chemical toner produced by a major manufacturer was used. To observe the internal components using TEM, a film-type specimen was prepared by an ultramicrotome (Powertome MT, RMC) at room temperature. Toner particles were first coated by the evaporation of a $2 \% \mathrm{RuO}_{4}$ solution for $15 \mathrm{~min}$. This process was performed to prevent the absorption of the toner in the embedding media (epoxy). Then, the particles were embedded in a mixture of epoxy and hardener. TEM systems operated at $200 \mathrm{keV}$ with a field-emission gun (Tecnai F20st, FEI) and $1.25 \mathrm{MeV}$ with a tungsten filament (JEM-ARM 1300S, JEOL) were used for 
microscopic observation from $-60^{\circ}$ to $+60^{\circ}$ with a $3^{\circ}$ tilt offset to the $x$-axis for Z-contrast and BF images. An electron energy-loss spectrometry (EELS) system incorporated in the UHV-TEM was used to measure inelastic scattering. The series of recorded micrographs were analyzed through a commercialized code, Amira, to reconstruct the internal distribution of wax.

\section{Results AND Discussion}

The image degradation of present meso-scale tomography was approached with respect to the geometry, such as DOF and beam divergence, with the electron scattering in the interaction volume. DOF defines the depth of sharpness and the distance along the beam axis within, in which the object can be moved without detectable loss of the image sharpness (Williams \& Carter, 1996). The DOF originates from a two-dimensional projection of the three-dimensional image formed by an objective lens. As for STEM, which uses a convergent beam, the initial probe size $(d)$ increases by the divergence angle for a thick specimen. The mean probe size $(R)$ can be measured from the given convergence angle $(\alpha)$ and initial probe size $(d)$ for a specimen thickness $(t)$, as shown in equation (2). Both are inversely proportional to the spatial resolution and must exceed the virtual specimen thickness with respect to the required resolution to form a qualified image recognizable by the tomography code. Equations for DOF and $R$ can be expressed as follows

$$
\begin{aligned}
\mathrm{DOF} & =d / \beta \\
R & =\left(d+R_{\max }\right) / 2=1.61 \lambda \sin ^{-1} \alpha+t \times \tan \alpha
\end{aligned}
$$

where $d$ is the distance corresponding to the smallest distances that can be resolved in the object, $\alpha$ and $\beta$ are the collection semi-angle with respect to the condenser and objective lens, and $\lambda$ 's for 200 and 1,250 keV equals $25.1 \times$ $10^{-4}$ and $7.354 \times 10^{-4} \mathrm{~nm}$, respectively.

In the consideration of electron scattering, beam spread (b) and energy spread contributing to the spatial resolution are limited to the real specimen volume. $\boldsymbol{b}$ is proportional to the atomic number $(Z), t^{3 / 2}$, and inversely proportional to electron beam energy $(E)$ as shown in equation (3). The energy spread causes chromatic aberration in that the objective lens deflects electrons of lower energy more strongly, and thus the electrons from a point in the sample form a disk image (Williams \& Carter, 1996). The radius $\left(r_{\mathrm{ch}}\right)$ of the disk proportional to the losses of energy $(\Delta E)$ maintains the relationship in equation (4). EELS estimated the energy spread, and the energy loss was converted to $r_{\mathrm{ch}}$ in Figure 1:

$$
\begin{gathered}
b \infty Z / E \times t^{3 / 2} \\
r_{\mathrm{ch}}=C_{\mathrm{c}}\left(\Delta E / E_{0}\right) \beta
\end{gathered}
$$

where $C_{\mathrm{c}}=0.41 \mathrm{~cm}$ is defined by the manufacturer and $E_{0}$ is the energy of incident electrons.

Good Z-contrast images were obtained from a toner section less than $\sim 4 \mu \mathrm{m}$ in the lateral radius, which was nearly within in-focus for the required magnification range

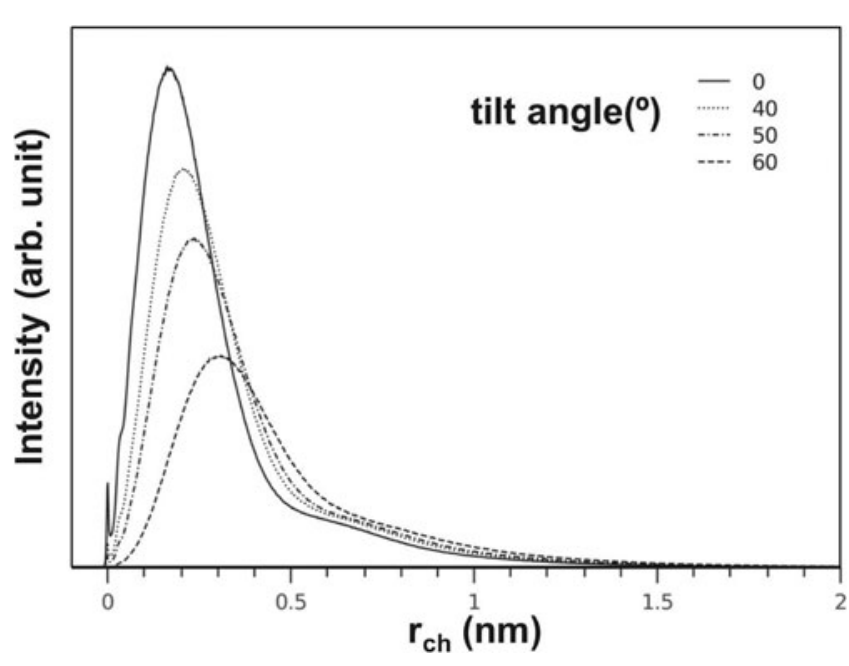

Figure 1. Distribution of $r_{\mathrm{ch}}$ with specimen tilting angle. $r_{\mathrm{ch}}$ corresponds to the energy loss $(0-1.5 \mathrm{keV})$ measured by electron energyloss spectrometry equipped to a $1.25-\mathrm{MeV}$ ultra-high-voltage transmission electron microscope. The effective specimen thickness increased from $1,500 \mathrm{~nm}\left(0^{\circ}\right.$ tilt $)$ to $1,732 \mathrm{~nm}\left(60^{\circ}\right.$ tilt $)$.
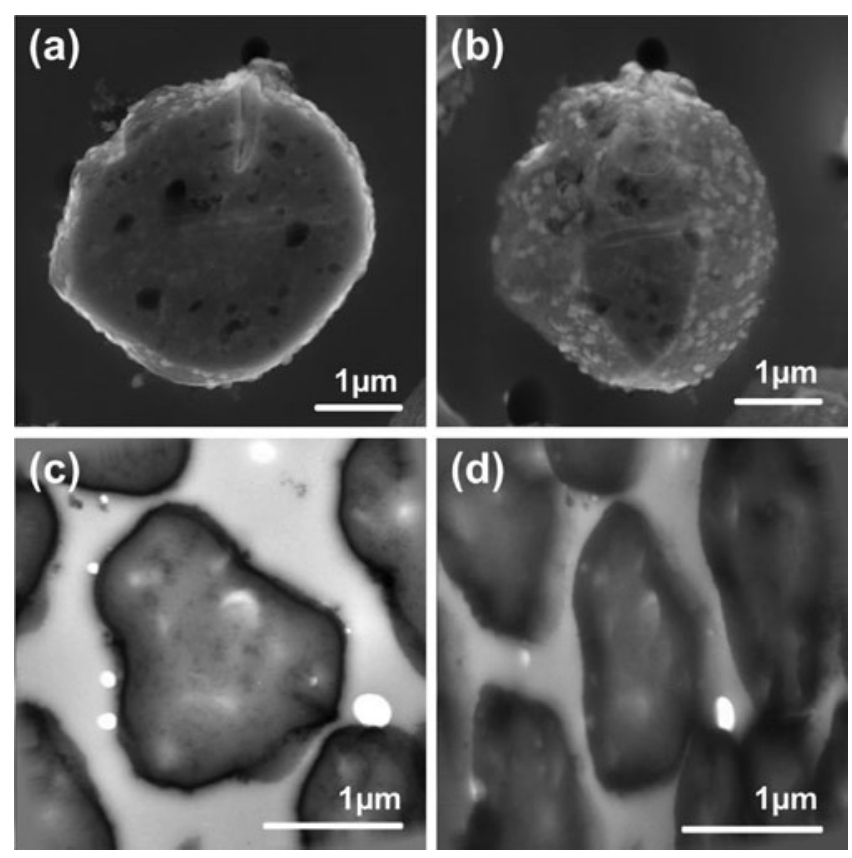

Figure 2. a, b: Z-contrast images captured with a $200-\mathrm{keV}$ scanning transmission electron microscope at the specimen tilt angle of 0 and $60^{\circ}$. c, d: Bright-field (BF) images captured with a 1.25$\mathrm{MeV}$ ultra-high-voltage TEM at the specimen tilt angle of 0 and $60^{\circ}$. The noticeable degradation of image quality was observed in $\mathrm{BF}$ image at high tilt angle.

of the STEM operated at $200 \mathrm{keV}$. Toner sections thicker than $4 \mu \mathrm{m}$ in the lateral radius exhibited obvious image degradation at a high tilt angle, which may have originated from the geometry. Figures $2 \mathrm{a}$ and $2 \mathrm{~b}$ show the $\mathrm{Z}$-contrast image at 0 and $60^{\circ}$ specimen tilt for the $\sim 4-\mu \mathrm{m}$ toner section. The image exhibits nearly similar noise over the whole region and clearly illustrates the surface topography of the sectioned toner with few $\mathrm{RuO}_{4}$ particles. The wax 
contrast was rather dark in the Z-contrast image. The shape of the small pigment particles was also clearly distinguished. The thickness of the toner section was measured $\sim 1,500 \mathrm{~nm}$ coincidentally from the tilt image and its reconstructed tomogram.

HAADF-STEM uses highly deflected ( $>50 \mathrm{mrad}$ ) electrons to form a Z-contrast image. As it is free from the objective lens, once the incident electron is scattered the probability of the secondary collision reduces greatly by a factor of $p^{2}$, where $p$ is the scattering probability. As a result, the contribution of beam spreading in the spatial resolution $(R)$ of the Z-contrast image is less than a BF image, which contains most of the scattered electrons. By using thickly sectioned polymer film $(\sim 1 \mu \mathrm{m})$, Hyun et al. (2008) illustrated that the spatial resolution of a Z-contrast image was dominated by beam divergence, and the beam spreading was negligible.

The contribution of DOF and beam divergence for image degradation increases greatly with specimen tilting. The required DOF $(1,500$ and 4,214 $\mathrm{nm}$ with respect to 0 and $\pm 60^{\circ}$ tilt) at a given $\alpha$ is proportional to $\sin \theta$, where $\theta$ is the tilt angle and $\alpha$ is $8 \mathrm{mrad}$ measured from a $70-\mu \mathrm{m}$ aperture. By applying the DOFs [equation (1)], $d$ 's are 12 and $34 \mathrm{~nm}$ for the 0 and $\pm 60^{\circ}$ tilt, respectively. The $R$ values determined by beam divergence and the initial probe size were $\sim 17$ and $\sim 38 \mathrm{~nm}$, respectively. The initial probe size was determined by the Raleigh criteria. However, DOF did not contribute to the degradation of the Z-contrast image. In STEM mode, each pixel of the Z-contrast image is acquired in a serial manner using the HAADF detector, as the electron probe scans the view area sequentially. This serial nature of the image acquisition in STEM makes the DOF completely different from the DOF appearing in the parallel recording (BF images) in conventional TEM. As a result, the spatial resolution for STEM precisely fits the beam divergence $(R)$ as in the experiment of Hyun et al. (2008).

In the case of the BF for a similar lateral-sized toner section (see Figs. 2c, 2d), application of the semi-convergence angle $(\beta), \sim 0.4 \mathrm{mrad}$, produces $d$ 's of 0.7 and $9 \mathrm{~nm}$ with respect to the required DOFs. Related to the electron scattering, energy spread was deduced from the EELS spectrum showing distribution of energy losses $(\Delta E)$ of the incident electrons. On applying equation (4) to the recorded EELS spectrum, the distribution of $r_{\mathrm{ch}}$ shows that the BF images were mostly formed of electrons in the energy loss range of $0-300 \mathrm{eV}$ (see Fig. 1). With increased tilt of the specimen, larger and broader energy loss compared with the real volume change occurred. Nevertheless, the change of $r_{\mathrm{ch}}$ restricted to $1.5 \mathrm{~nm}$ was not a dominant factor for the image degradation compared with the $d$ values measured in the STEM. $\boldsymbol{b}$ was nearly negligible based on the Monte Carlo simulation using the code CASINO with respect to 0 and $60^{\circ}$ tilt (Hovington et al., 1997). Styrene-acrylate copolymer was used for simulation, which occupies $80-90 \mathrm{wt} \%$ of the toner with a density of $\sim 1.1 \mathrm{~g} / \mathrm{cm}$ (Kazuo et al., 1991; Michael et al., 2011). The beam spread from multiple scat-
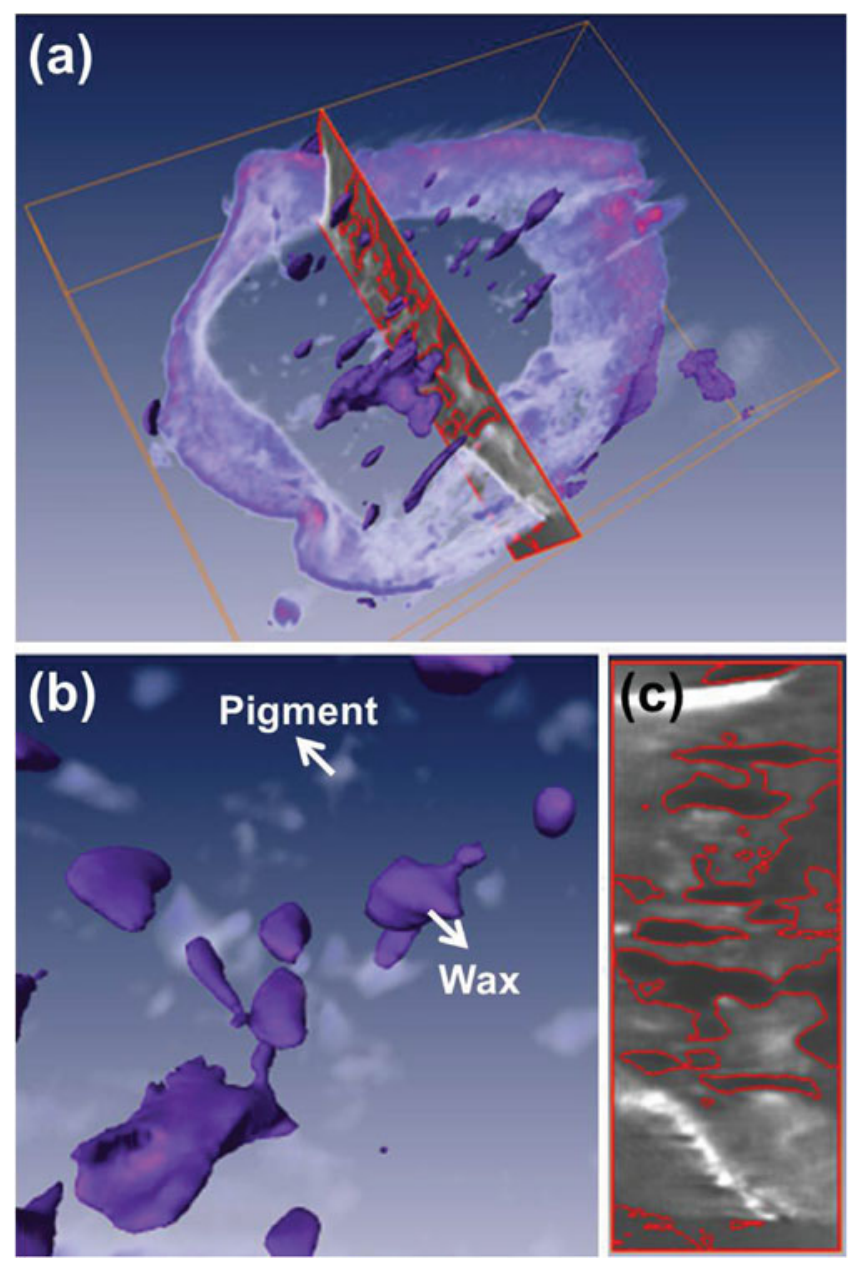

Figure 3. a: Reconstructed tomogram using a series of Z-contrast images acquired with a $200-\mathrm{keV}$ scanning transmission electron microscope. The distribution of wax and even the smaller pigment is clearly reconstructed. b: Enlarged image of (a) showing clear distinction between the wax and the small pigment. c: The intensity map of the cross-sectioned area of the rectangle in the tomogram (a). Successive image rendering gives a color to a range of the intensity of (c) such as low, medium, and high intensity to resin, wax, and pigment in (a), respectively.

terings produced degradation in the Z-contrast image. Equation (3) for UHV produces a six times smaller $\boldsymbol{b}$ than that of $200 \mathrm{keV}$. The $\boldsymbol{b}$ seems to consist of single scattered electrons small enough for the Z-contrast images, confirming the simulation result. Therefore, the simulated $\boldsymbol{b}$ for UHV could be real. Similarly, a previous report also produced a negligible $\boldsymbol{b}$ for an $\sim 1-\mu \mathrm{m}$-thick polymer section (Hyun et al., 2008).

Conclusively, the degradation was mainly caused by beam divergence for the Z-contrast image and by the combination of DOF and $C_{\mathrm{c}}$ for the BF image of UHV-TEM. Additional studies will determine the final spatial resolution from the combination of DOF and $r_{\mathrm{ch}}$, as the quality was greater in the Z-contrast image in the medium-voltage TEM than the BF image in UHV-TEM. This difference contributes to the failure in $\mathrm{BF}$ tomogram. As a result, the degradation of the acquired $\mathrm{BF}$ images produced recogni- 
tion errors by Amira. In contrast, a tomogram was successfully reconstructed from the series of Z-contrast images as shown in Figure 3. Moreover, small contrasts between wax, resin, and pigment in the BF mode were enhanced, as the Z-contrast was proportional to $Z^{1.7}$ (Kirkland et al., 1987). The scale of Z-dependent contrast for BF-TEM is known as $\sim Z^{0.75}$ (Kübel et al., 2005). Tomography simply required clear contrast (high signal-to-noise ratio) with high intensity to construct attenuation function according to the viewing angle and position (Friedricha et al., 2005). The resultant tomogram of the Z-contrast image clearly reconstructed the distribution of wax and even the smaller pigment, as shown in Figure 3.

\section{SUMMARY}

We have successfully visualized the three-dimensional distribution of wax in toner using medium-voltage STEM rather than UHV-TEM with the aid of tomography. We briefly compared geometrical effects, such as DOF and beam divergence, with the beam-specimen interaction of $\boldsymbol{b}$ and $C_{\mathrm{c}}$. The failure in tomogram reconstruction of UHV-BF was primarily due to the combination of DOF and $C_{\mathrm{c}}$. The very short wavelength of UHV-TEM greatly minimized DOF, $\mathrm{r}_{\mathrm{ch}}$, and $\boldsymbol{b}$. Nevertheless, it had insufficient quality for the thick polymer $(\sim 1.5 \mu \mathrm{m})$ tomography. The spatial resolution of STEM governed solely by $R$ was sufficient to differentiate the internal structure of the wax and even smaller pigment. The superiority of STEM at a medium voltage could be applied to the meso-scale tomography of low $\mathrm{Z}$ materials, such as polymers.

\section{ACKNOWLEDGEMENTS}

The authors thank Dr. Kim Goo-Myun and his co-workers (Samsung Fine Chemical Co.) for the discussion on the properties of toner. The authors are especially grateful to Dr. Yon Kyung-Yol for providing us with the motivation to conduct the present study. This work was supported by the National Research Foundation of Korea (NRF) grants funded by the Korea government (MEST; No. 2011-0017257, No. 2011-0019984, and No. 2011-0030803).

\section{REFERENCES}

Cosslett, V.E. (1979). Penetration and resolution of STEM and CTEM in amorphous and polycrystalline materials. Physica Status Solidi (a) 55, 545-548.

Friedricha, H., McCartney, M.R. \& Busecka, P.R. (2005). Comparison of intensity distributions in tomograms from BF TEM, ADF STEM, HAADF STEM, and calculated tilt series. Ultramicroscopy 106, 18-27.

Hovington, P., Drouin, D. \& Gauvin, R. (1997). CASINO: A new Monte Carlo code in C language for electron beam interactionspart I: Description of the program. Scanning 19, 1-14.

Hyun, J.K., Ercius, P. \& Muller, D.A. (2008). Beam spreading and spatial resolution in thick organic specimens. Ultramicroscopy $109,1-7$.

Kazuo, T., Shinichi, K., Kazuhiko, U. \& Hidemi, U. (1991). Toner for electrophotography including fluorine contained graft copolymer. U.S. Patent 5,061,587.

Kirkland, E.J., Loane, R.F. \& Silcox, J. (1987). Simulation of annular dark field stem images using a modified multislice method. Ultramicroscopy 23, 77-96.

Kübel, C., Voigt, A., Schoenmakers, R., Otten, M., Su, D., Tan-Chen, L., Carlsson, A. \& Bradley, J. (2005). Recent advances in electron tomography: TEM and HAADF-STEM tomography for materials science and semiconductor applications. Microsc Microanal 11, 378-400.

Maruta, M. (1997). The material design of the polyester color toners. In Recent Progress in Toner Technologies, Marshall, G. (Ed.), pp. 126-130. Springfield: IS\&T.

Michael, B.C., Aura, B.L., James, B.M., Joseph, K.J., Matthew, T.M. \& JING, X.S. (2011). Polyester resin toner produced by emulsion aggregation. U.S. Patent 7,923,191 B2.

Williams, D.B. \& Carter, C.B. (1996). Transmission Electron Microscopy. New York: Plenum. 